Voix et Images

voixetimages

\title{
Éloge de la fiction
}

\section{Pierre Hébert}

Volume 17, numéro 3 (51), printemps 1992

Paul-Marie Lapointe

URI : https://id.erudit.org/iderudit/200985ar

DOI : https://doi.org/10.7202/200985ar

Aller au sommaire du numéro

\section{Éditeur(s)}

Université du Québec à Montréal

\section{ISSN}

0318-9201 (imprimé)

1705-933X (numérique)

Découvrir la revue

Citer cet article

Hébert, P. (1992). Éloge de la fiction. Voix et Images, 17(3), 529-535.

https://doi.org/10.7202/200985ar

Ce document est protégé par la loi sur le droit d'auteur. L'utilisation des services d'Érudit (y compris la reproduction) est assujettie à sa politique d'utilisation que vous pouvez consulter en ligne.

https://apropos.erudit.org/fr/usagers/politique-dutilisation/
Cet article est diffusé et préservé par Érudit.

Érudit est un consortium interuniversitaire sans but lucratif composé de l’Université de Montréal, l'Université Laval et l'Université du Québec à Montréal. Il a pour mission la promotion et la valorisation de la recherche. https://www.erudit.org/fr/ 
Roman

\section{Éloge de la fiction}

Pierre Hébert, Université de Sherbrooke

Il constata que la vie n'était qu'une série de questions sensées qui avaient une réponse absurde.

Robert Gurik, Être ou ne pas être, p. 154

Au fond, la vie n'est belle qu'inventée.

François Piazza, Les Valseuses du Plateau Mont-Royal, p. 60 
L'absurde - Albert Camus, vous vous souvenez? - n'est ni dans l'homme, ni dans le monde; il se situe plutôt entre les deux, l'homme interrogeant le monde et celui-ci se taisant à propos des questions essentielles. Cette rupture entre l'humain et son univers est véritablement la patrie de l'absurde: j'interroge désespérément un monde qui se tait. Jamais, jamais mes interrogations n'obtiendront de réponses certaines. L'absurde c'est, disait Camus, ce cri immémorial de millions d'êtres humains qui se perd dans le silence déraisonnable du monde.

Déraisonnable, ce silence du monde? Prenons alors le mot au pied de la lettre, et allons voir ailleurs si nous y sommes. Allons voir du côté de la fiction. Car n'est-ce pas elle, la fiction, qui s'insère dans ce petit espace de rupture qu'il y a entre soi et le mondé? Mais, au contraire de la raison, elle n'a de cesse qu'elle nous ait troublés, malmenés au point de nous pousser à imaginer une vie tout autre que celle qui, maintenant, ne nous satisfait pas. Au slogan de naguère des forces armées - "Si la vie vous intéresse! - - opposons celui de la fiction: si la vie ne vous suffit' pas!

Car la fiction ne quête pas l'intelligible mais plutôt; on le sait bien, l'idéal, voire l'utopique. L'état second que fait entrevoir la vie écrite n'est jamais "réaliste", si bien qu'il comporte aussi des dangers qu'un correspondant de la Gazette de Québec de 1770 (oui, 1770!) avait bien compris en établissant un lien entre le malheur de certains mariages et... le théâtre ou le roman! Il écrivait ce qui suit:

La vérité est que nous faisons l'amour avec un stile, et des sentiments peu convenables à la vie ordinaire; ils sont presque conformes au théâtre et aux romans. Par ce moien nous élevons notre imagination à quoi on ne doit pas s'attendre dans la vie humaine [...].

La seule façon d'être heureux, poursuit-il, est de "considérer les choses dans leur vrai jour, et comme la nature les a formés, et non comme notre imagination et nos désirs voudroient qu'elles fussent . Ce correspondant avait-il lu Pascal? La littérature est ainsi une espèce aberrante de l'état naturel.

Dans cet excellent recueil de nouvelles de Lori Saint-Martin, Lettre imaginaire à la femme de mon amant ${ }^{1}$, ce qui m'a justement frappé, outre la qualité, la concision de l'écriture, c'est comment l'auteure fait jouer à plein le pouvoir de la fiction, de l'imaginaire. Certes, le titre déjà l'annonce, qui est celui de la seconde nouvelle du texte: ‘Imaginaire, cette lettre, parce que je ne veux pas te connaitre ni, surtout, que tu me connaisses. (p. 9), écrit la mâitresse d'André à sa femme Caroline. Elle en profite, certes, pour faire le point sur leur vie, mais 
aussi pour rassurer la femme trompée: *Autant que cette lettre, je suis imaginaire et le demeurerai." (p. 16)

Elle évolue tout autant dans la vie fictive cette femme qui écrit: «Je n'ai pas d'amants, je trompe mon mari avec ce cahier.» (p. 47) «Sur fond de rupture" est une nouvelle qui consacre le pouvoir subordonnant de la fiction où l'écriture conduit une femme à se sauver de son mari et à vivre seule: "Lorsque le jour sera venu, je me ferai toute petite et je rentrerai dans mon cahier pour de bon. Aucun lieu sur cette terre ne me paraît aussi attirant.. (p. 51)

Comment un plus un peuvent-ils donner trois? Encore par la fiction: ainsi cette autre femme qui trompe son mari et qui est obligée de s'inventer un personnage. Deux vies réelles, avec le mari et l'amant, mais une troisième vie, fictive, pour maintenir les deux premières:

Avoir une double vie n'est justement pas simple, il faut de l'énergie,' des calculs, un cœur solide. Car pour mener une double vie, il en faut vraiment trois. Aux deux qu'on vit - l'officielle et la secrète - s'ajoute celle qui justifie les absences nécessaires à l'amour. Celle-là on l'invente, simple récit. Et puis tout d'un coup elle se met à gonfler, à prendre toute la place, au risque d'éclipser toutes les autres. Récits pour me donner du temps, fragiles récifs. (p. 34)

Récits, récifs; recueil, écueil: la vie imaginaire est souvent dangereuse, fragile. Lettre imaginaire à la femme de mon amant est plein de ruptures, à la manière de cette superbe photographie en page couverture d'un avocat coupé en deux: le fruit est scindé, mais le noyau reste entier.

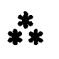

Le titre même du recueil de nouvelles de Robert Gurik, Etre ou ne pas être ${ }^{2}$, est grave; et ses textes le sont tout également. Chacun pose des situations qui interrogent à la fois le sens de la vie, de la mort et du bonheur, de même que les valeurs de notre société actuelle. Oscillant sans' cesse entre deux tableaux contraires, le recueil aurait très bien pu s'appeler *Être et né pas être.

C'est néanmoins la question de Hamlet qui prévaut ici; mais, sans écarter son sens métaphysique, sa résonance est le plus souvent sociale. Chacune des six nouvelles fait à l'occasion des clins d'œil à sa voisine, et toutes font converger leurs propos autour de la vie, de la qualité de la vie plus précisément. George, dans la nouvelle «Le pain 
de tout le monde", pose ainsi la question qui hante plusieurs personnages: qu'est-ce qui vaut le mieux, "une vie abrégée ou une mort élonguée?* (p. 70) Est-ce que le plus important *c'est de sauver sa peau, conserver ce qu'on a, vivre le plus longtemps possiblem.? (p. 78)

La première nouvelle, "L'œil »; met en scène Paul qui, peu à peu, est envahi par une étrange maladie: une forme d'abord floue, mais de plus en plus précise, hante l'intérieur de son œil. Tigre, gorille, puis dame à la faux, cette forme entraîne Paul à chercher, à l'âge de soixante ans, l'essentiel de la vie: ne pas souffrir. La forme le harcèlé sur ce qu'il veut encore de la vie ou, plus justement, peut encore:

Un bon repas? Tu n'as plus l'estomac pour le digérer. Une belle jeune femme? Ton corps flasque et vieilli te fait honte. Un bon livre, un bon spectacle? Il t'arrive maintenant de t'endormir en plein milieu. L'action sociale politique? Même jeune, tu n'as rien fait et en plus tout ce que tu hais est revenu. Moi, à ta place... (p. 10)

Prélude à la mort, la tentation qui s'offre à George est celle du désespoir. Nuançons: celle de ne plus rien espérer. En écho au lointain Alfred de Vigny qui, dans son Journal d'un poète, 'écrivait que l'espoir est la plus grande de nos folies, Paul l'obsédé se fait dire par la forme qui parle que "l'homme heureux est celui qui n'a plus rien à espérer. Espérer, c'est attendre. Le vrai bonheur commence quand on n'attend plus." (p. 17) Que reste-t-il d'autre à faire que de quitter la vie, de sortir de ce qu'un autre personnage appellera "le mouroir" (p. 84)?

En parallèle avec ce dilemme de la vie abrégée et de la vie élonguée, la fiction joue un rôle déterminant dans chacune des nouvelles. Il y a Paul qui "se dit qu'il était fou de prendre une ombre pour la réalité encore plus fou de prendre la réalité pour la réalité» (p. 13); George, qui se sent soulagé lorsque, pour la première fois, il réussit à écrire le malaise qu'il a toujours ressenti; mais surtout Germain, qui visite sa mère hospitalisée:

Après ses crises de paranoïa, de sabbat infernal et de tentatives d'empoisonnement, elle confiait à Germain que, pour meubler son ennui, elle inventait des histoires, des - romans * et que : peut-être je confonds mes inventions avec la réalité.

Germain se demanda où était la frontière entre les inventions cauchemardesques de sa mère, qui devenaient pour elle réalité et dont elle souffrait, et la réalité qui devenait pour lui matériel de fiction? (p. 28)

Être ou ne pas être est un recueil qui frappe par l'originalité de son discours. De ses discours, devrait-on dire, le pluriel étant de rigueur. Car s'entremêlent ici, dans le bon sens du mot, le réalisme, le 
fantastique et une manière de fiction prospective qui ont pour sauce liante un discours social et métaphysique dérangeants. Toute cette artillerie discursive est cependant nécessaire, car "l'homme, comme la femme, est un animal étrange qu'aucun être humain ne pourra comprendre complètement $=($ p. 111).

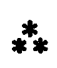

Sarabande: danse populaire espagnole de mouvement plutôt lent, nous apprend le dictionnaire. C'est le titre du roman de Guylène Saucier ${ }^{3}$, c'est aussi ce que joue le personnage principal:

Élise avait joué. Le remous de la conversation s'était éteint tout seul. Une sarabande de Haendel, triste, lente et grave. Son visage était devenu pâle comme la cire. On aurait dit une colombe posée sur un manteau noir. Elle jouait ici la fin de sa'vie. Les dernières notes. Elle n'arrivait pas à les toucher, ses doigts étaient restés suspendus au-dessus du clavier, puis lorsqu'ils avaient plaqué l'accord, aucun son n'était monté. (p. 78)

Mais qui est Élise? Il s'agit d'une jeune femme, disparue mystérieusement la nuit du 31 mars 1976. Le récit recourt à une technique bien connue, qui consiste à faire varier le point de vue à chaque chapitre; ainsi se succèdent Monsieur George, le propriétaire de motel où se tenait Élise, William Ruben, l'amant américain, Antoine Vanasse, le mari, Jeanne, la sœur et, bien sûr, Élise elle-même: Le récit n'est pas sans rappeler Les Fous de Bassan, d'Anne Hébert, par cette mosaïque de perspectives autour d'une disparition.

Les ressources de la fiction sont utilisées ici pour une autre fin, celle d'activer l'imaginaire dans la reconstitution non seulement de l'intrigue, mais aussi, mais surtout dans la composition du personnage même d'Élise. Cette approche du personnage s'apparente, mutatis mutandis, à celle du roman poétique où l'être n'est pas donné mais construit, et où il est beaucoup plus un effet de langage que le résultat d'une fonction. Certes la disparition mystérieuse du 31 mars appelle une solution, mais ce faire est subordonné à l'être du personnage.

La fiction s'efforce ainsi de combler l'absence: "Toutes les images que j'ai d'elle [Élise], je les possède, dit Monsieur Georges. Je sais qu'elles ne sont pas les seules qui existent, mais les miennes je les connais par coeur et je les adore.» (p. 115) Élise se réduit, du point de vue des autres personnages, à des notations de souvenirs et d'images mais toutes n'ont pas le caractère positif de celles de Monsieur Georges: "Maintenant, avoue William Ruben, je ne vois que le temps 
qui m'a échappé, et cette fille, et l'idée fausse que je m'en suis faite.* (p. 129-130)

Sarabande a plusieurs qualités; mais sa plus belle est peut-être son unité de vision, malgré quelques dissonances de discours, autour $\mathrm{du}$ personnage principal. Jaillissant avec aisance et élégance d'un foyer commun, l'écriture sert l'intrigue mais, avant tout, avec le naturel de la lumière, elle laisse progressivement voir son objet sans se faire voir elle-même.

"Mon grand-père détestait les romans. Il disait que ce n'était pas la vie, que ce n'était que des mensonges. Alors si l'on vous prenait avec un roman dans les mains, vous étiez bon pour la corde ${ }^{4}$." Dany Laferrière, lui, n'a certainement pas suivi les conseils de son aïeul, sinon il n'eût pas écrit L'Odeur du café. Car pour avoir écrit ce récit, il fallait croire à autre chose qu'aux mensonges de la fiction.

En vérité, cette troisième œuvre de Dany Laferrière s'apparente beaucoup plus à des souvenirs qu'à un récit. Les souvenirs, on le sait, présentent une allure généralement décousue, leur unité se trouvant, parfois, dans l'époque qu'ils évoquent ou encore le lieu de leur déroulement. Cette allure brisée du texte, Dany Laferrière l'utilise amplement, faisant précéder plusieurs segments, jusqu'à deux ou trois par pages, d'un sous-titre. Ce qui est raconté ici, c'est l'enfance du narrateur (il a dix ans en 1963) à Petit-Goâve, non loin de Port-auPrince. Le narrateur déplie des souvenirs d'enfance, des images lointaines qui l'ont hanté jusque dans son présent, quelquies trente années plus tard. L'Odeur du café ne raconte pas vraiment; -il peint, dessine, évoque.

Ce genre "cahier-spicilège " permet d'introduire, sans nuire au ton de l'ensemble, une variété d'énoncés disparates. Ici, vous avez bien sûr le portrait d'un personnage; là, une sorte de maxime qui conclut une séquence ( $\mathrm{Da}$ - sa grand-mère - dit que c'est comme ça qu'on reconnaît les riches, ils peuvent laisser les fruits pourrir au sol. [p. 45]). Parfois, c'est l'ensemble du micro-récit qui prend une valeur exemplaire ou, encore, c'est un rêve qui a besoin d'une interprétation.

La fiction sert donc ici un nouveau maître: l'évocation du passé, la notation d'une collection d'images pour la plupart paisibles. Les gens heureux n'ont peut-être pas d'histoire, mais ils ont des souvenirs. 
Combien de romans se publie-t-il chaque année au Québec? Cent cinquante? Deux cents? Quel qu'en soit le nombre exact, une chose est certaine: cette quantité effarante a de quoi donner, disons à un chroniqueur par exemple, des sueurs froides. Car comment choisir quand on ne peut tout lire? Comment rédiger une chronique vaguement thématique sans que les liens faits entre les œuvres ne soient pas eux-mêmes pure fiction? Et combien de ces œuvres, diront les gens réalistes, ne valaient de toutes façons pas la peine d'être publiées, ni même d'être écrites?

C'est donc avec perṕlexité que je regarde, sur la tablette de ma bibliothèque, les quelque soixante-quinze romans qui composent "mon * année 1991. Mais je me dis aussi que ce qu'il ne faut jamais oublier, c'est que chacune de ces œuvres, quelle que soit sa *valeur"; participe à nous dégager de la gangue trop étroite du réel. La création s'écarte de la pente naturelle de la raison: il n'y a donc pas seulement, quoi qu'en ait pensé Camus, des interrogations humaines restées sans réponses; il y a aussi chacun de ces mondes inventés à mesure de rêve. Hélas! la portée politique du pouvoir d'imaginer n'a pas encore été pleinement comprise! L'éloge de la fiction devrait ainsi tenir compte de toutes les voix qui la composent. En guise de dernier texte, le chroniqueur rend donc hommage à toutes celles et tout ceux qui, dans le silence de leur bibliothèque, dans l'isolement de leur salle de travail, ont tenté d'échapper aux forces de la gravité terrestre.

1. Lori Saint-Martin, Lettre imaginaire à la femme de mon amant, Montréal, L'Hexagone, 1991, $135 \mathrm{p}$.

2. Robert Gurik, Etre ou ne pas être, Montréal, XYZ éditeur, coll. :L'Ère nouvelle, 1991, 173 p.

3. Guylaine Saucier, Sarabande, Montréal, Québec/Amérique, 1991, 166 p.

4. Dany Laferrière, L'Odeur du café, VLB éditeur, Montréal, 1991, 200 p. (cf. p. 28). 